\title{
Nonlinear Relationship Between HbA1c and Coronary Artery Calcium Score Progression: A Secondary Analysis Based on a Retrospective Cohort Study
}

Jing Yu

Guizhou Medical University

Bo Gao ( $\sim$ gygb2004@163.com )

Guizhou Medical University

\section{Research}

Keywords: Glycated hemoglobin, coronary artery calcium score, Coronary atherosclerotic heart disease

Posted Date: May 13th, 2021

DOl: https://doi.org/10.21203/rs.3.rs-505751/v1

License: (c) (1) This work is licensed under a Creative Commons Attribution 4.0 International License. Read Full License

Version of Record: A version of this preprint was published at Diabetology \& Metabolic Syndrome on November 19th, 2021. See the published version at https://doi.org/10.1186/s13098-021-00747-z. 


\section{Abstract}

Objective

Some previous studies was to clarify the correlation between glycated hemoglobin $(\mathrm{HbA} 1 \mathrm{c})$ and coronary heart disease (CHD) and the evidence regarding the correlation was still debated. However, there are fewer scientific dissertations about the correlation between $\mathrm{HbA} 1 \mathrm{c}$ and coronary artery calcium score progression. Consequently, the present study was undertaken to explore the link of $\mathrm{HbA} 1 \mathrm{c}$ on coronary artery calcium score progression in South Korea.

\section{Methods}

This study is a secondary analysis based on a retrospective cohort study. 8151 participants received a health check-up program at the Health Promotion Center of the Samsung Medical Center in Seoul, South Korea, from March 1, 2003 to December 31, 2013. We then used Cox proportional-hazards regression model to evaluate the independent relationship between $\mathrm{HbA} 1 \mathrm{c}$ and coronary artery calcium score progression.

Results

After adjusting potential confounders (age, sex, BMI, height, weight, SBP, DBP, TC, LDL-C, HDL-C, triglycerides,smoking status, alcohol consumption, reflux esophagitis status, hypertension, diabetes, dyslipidemia, ischemic heart disease and cerebrovascular disease), non-linear relationship was detected between $\mathrm{HbA} 1 \mathrm{c}$ and coronary artery calcium score progression, whose point was $5.8 \%$. The effect sizes and the confidence intervals on the left and right sides of inflection point were 2.05 (1.85 to 2.27) and 1.04 (0.99 to 1.10$)$, respectively.

Conclusion

The relationship between $\mathrm{HbA} 1 \mathrm{c}$ and coronary artery calcium score progression is non-linear. $\mathrm{HbA} 1 \mathrm{c}$ is positively related with coronary artery calcium score progression when $\mathrm{HbA} 1 \mathrm{c}$ was less than $5.8 \%$.

\section{Introduction}

Glycated hemoglobin ( $\mathrm{HbA1c}$ ) is the core of glycemic control and management in diabetic patients, and has recently been recommended for diagnosing diabetes and identifying people at risk of developing diabetes[1]. Compared with fasting or post-load blood glucose measurement, $\mathrm{HbA1} \mathrm{c}$ has better reliability[2]. HbA1c can effectively reflect blood glucose control in the past 2-3 months, and is a diagnosis and recommendation for diabetes screening tool[3-4].

Coronary atherosclerotic heart disease (coronary heart disease for short, CHD) is a multi-cause disease. The Framingham Heart Study in the United States has identified a series of risk factors for coronary heart disease, including age, gender, cholesterol, hypertension, smoking and diabetes[5]. It is well known that 
chronic glucose metabolism disorders increase the risk of $\mathrm{CHD}[6-9]$. However, many previous studies regarding the association between $\mathrm{HbA} 1 \mathrm{c}$ and $\mathrm{CHD}$ yielded conflicting findings. Many studies reported that $\mathrm{HbA} 1 \mathrm{c}$ was an important determinant of $\mathrm{CHD}$ and its severity[10-14]. However, Other researches suggested that the association between $\mathrm{HbA} 1 \mathrm{c}$ and $\mathrm{CHD}$ was not significant[15], and it was controversial whether it was necessary to control the patient's $\mathrm{HbA} 1 \mathrm{c}$ below $7 \%$ or even close to the normal level[16]. In view of the differences in study population, study design, measurement (define) of coronary artery stiffness, adjustment for covariates, and certain methodological limitations, we conducted a secondary analysis based on a retrospective cohort study to observe the correlation between $\mathrm{HbA} 1 \mathrm{c}$ and the progression of coronary artery calcium scores in people undergoing physical examinations at the Health Promotion Center.

\section{Participants And Methods Study design}

This research is a retrospective cohort study design. The interesting independent variable in the present work is $\mathrm{HbA} 1 \mathrm{c}$. The dependent variable is coronary artery calcium score progression ( dichotomous variable : 0 = no progression, 1 = progression).

\section{Data source}

We obtained data from the "PLOS ONE" database (https://journals.plos.org/plosone/). This website allowed users to download raw data for free. According to PLOS ONE Terms of Service, we cited PLOS ONE data package in the present study. (PLOS ONE data package: Min YW, Song BG, Kim HS, Kim K, Lee H, Min BH, Lee JH, Son HJ, Rhee PL, Kim JJ (2017) Data from: Associations between reflux esophagitis and the progression of coronary artery calcification: A cohort study. https://doi.org/10.1371/journal.pone.0184996.s001).

\section{Study population}

Yang Won Min et al.[17] completed the entire study. In order to allow to understand the entire research process more clearly, we have outlined the steps of the study here. The specific details are described in the original, reported by Yang Won Min et al. They non-selectively and consecutively collected persons who had undergone physical examinations at the Health Promotion Center of the Samsung Medical Center in Seoul, South Korea, from March 1, 2003 to December 31, 2013. To ensure the privacy of participants, Yang Won Min et al. encoded their identity information as the non-traceable codes. Clinical data were extracted from hospital electronic medical record system.

Initially, 199375 participants were initially involved in this study; 191224 participants were subsequently excluded from this study, leaving 8151 cases for the final data analysis (see flowchart for details, Fig. 1). Inclusion criteria included: (1) Participants were aged over 20 years and underwent health screening examinations;(2) Participants underwent screening esophagogastroduodenoscopy (EGD) and coronary computed tomography (CT) scanning during the same visit. Participants who did not undergo follow-up 
coronary CT scans $(n=19,038)$ and had missing information $(n=89)$ were excluded. We also excluded participants who were missing HbA1c at the first physical examination $(n=70)$. The author has detailed ethics and informed consent in previously published articles[17].

\section{Variables}

\section{HbA1c}

The information of $\mathrm{HbA1c}$ were obtained at baseline and were recorded as continuous variable.

\section{Coronary artery calcium score progression}

Our interesting outcome variable was coronary artery calcium score progression (dichotomous variable : $0=$ no progression, $1=$ progression). Brilliance 40 (Philips Medical Systems, Cleveland, Ohio), VCT LightSpeed 64 (GE Healthcare, Milwaukee, Wisconsin), or Discovery 750HD (GE Healthcare) multidetector CT scanners were used to acquire images of CAC. The scans were analyzed using Extended Brilliance Workspace (Philips Medical Systems) or Advantage (GE Healthcare) workstations. The CAC scores were calculated as described by Agatston et al. [18].

\section{Covariates}

In this study, the selection of covariates was based on published literature and our clinical experience. Based on the above principles, the following variables were used as covariates: (1) continuous variables: age, duration, body mass index (BMI), height, weight, systolic blood pressure (SBP), diastolic blood pressure (DBP), diastolic blood pressure (TC), low-density lipoprotein cholesterol (LDL-C), high-density lipoprotein cholestero (HDL-C), triglycerides; (2) categorical variables: sex (male, female), smoking status (never, past smoker, current smoker), alcohol consumption (never, past drinker, current drinker), reflux esophagitis status (negative, positive), hypertension (negative, positive), diabetes (negative, positive), dyslipidemia (negative, positive), ischemic heart disease (negative, positive), cerebrovascular disease (negative, positive).

\section{Statistical analysis}

Data are indicated as mean \pm standard deviation (normal distribution) or median (min,max)(skewed distribution) for continuous variables and as numbers and percentages for categorical variables. $\chi^{2}$ (categorical variables), student $\mathrm{T}$ test (normal distribution), or Man Whitney $\mathrm{U}$ test (skewed distribution) were used to detect the differences among different coronary artery calcium score progression (binary variable). To examine the association between $\mathrm{HbA} 1 \mathrm{c}$ and coronary artery calcium score progression, we constructed three distinct models using univariate and multivariate Cox proportional-hazards regression model, including non-adjusted model (no covariates were adjusted), minimally-adjusted model (only sociodemographic variables were adjusted) and fully-adjusted model (covariates presented in Table 1 were adjusted). Effect sizes with 95\% confidence intervals were recorded. Because Cox proportionalhazards regression model based methods are often suspected for their inability to deal with non-linear 
models. For that reason, nonlinearity between $\mathrm{HbA} 1 \mathrm{c}$ and coronary artery calcium score progression were addressed using Cox proportional hazards regression model with cubic spline functions and the smooth curve fitting (penalized spline method). If nonlinearity was detected, we firstly calculated the inflection point using recursive algorithm, and then constructed a two-piecewise Cox proportional-hazards regression model on both sides of the inflection point. 
Table 1

Baseline characteristics of participants $(N=8151)$

coronary artery calcium score progression

\begin{tabular}{|c|c|c|c|}
\hline & NO & YES & P-value \\
\hline $\mathrm{N}$ & 3050 & 5101 & \\
\hline HbA1c, mean \pm sd, $(\%)$ & $5.50 \pm 0.60$ & $5.73 \pm 0.82$ & $<0.001$ \\
\hline Sex, $(n, \%)$ & & & 0.046 \\
\hline Male & $2750(90.16 \%)$ & $4666(91.47 \%)$ & \\
\hline Female & $300(9.84 \%)$ & $435(8.53 \%)$ & \\
\hline Age group, $(n, \%)$ & & & $<0.001$ \\
\hline$<65$ & $2914(95.54 \%)$ & 4417 (86.59\%) & \\
\hline$>=65$ & $136(4.46 \%)$ & $684(13.41 \%)$ & \\
\hline Duration & $3.72 \pm 2.02$ & $3.97 \pm 2.23$ & $<0.001$ \\
\hline $\mathrm{BMI}$, mean $\pm \mathrm{sd},\left(\mathrm{kg} / \mathrm{m}^{2}\right)$ & $24.40 \pm 2.48$ & $24.93 \pm 2.59$ & $<0.001$ \\
\hline Height, mean \pm sd, $(\mathrm{cm})$ & $169.91 \pm 6.73$ & $169.15 \pm 6.62$ & $<0.001$ \\
\hline Weight, mean $\pm \mathrm{sd},(\mathrm{kg})$ & $70.59 \pm 9.37$ & $71.46 \pm 9.50$ & $<0.001$ \\
\hline $\mathrm{SBP}$, mean $\pm \mathrm{sd},(\mathrm{mmHg})$ & $117.41 \pm 15.14$ & $120.57 \pm 15.77$ & $<0.001$ \\
\hline $\mathrm{DBP}$, mean $\pm \mathrm{sd},(\mathrm{mmHg})$ & $74.76 \pm 10.50$ & $76.27 \pm 10.52$ & $<0.001$ \\
\hline $\mathrm{TC}$, mean $\pm \mathrm{sd},(\mathrm{mg} / \mathrm{dL})$ & $197.31 \pm 33.01$ & $199.34 \pm 34.79$ & 0.009 \\
\hline LDL-C, mean $\pm \mathrm{sd},(\mathrm{mg} / \mathrm{dL})$ & $127.30 \pm 30.06$ & $128.90 \pm 31.46$ & 0.024 \\
\hline HDL-C, mean $\pm \mathrm{sd},(\mathrm{mg} / \mathrm{dL})$ & $52.61 \pm 12.86$ & $51.41 \pm 12.55$ & $<0.001$ \\
\hline Triglycerides, median (Q1-Q3), (mg/dL) & $121.00(86.00-171.00)$ & $150.58 \pm 90.51$ & $<0.001$ \\
\hline Smoking status, (n, \%) & & & $<0.001$ \\
\hline Never & $885(34.25 \%)$ & $1276(28.62 \%)$ & \\
\hline Past smoker & $916(35.45 \%)$ & $1837(41.21 \%)$ & \\
\hline Current smoker & $783(30.30 \%)$ & $1345(30.17 \%)$ & \\
\hline Alcohol consumption, $(\mathrm{n}, \%)$ & & & 0.018 \\
\hline
\end{tabular}

$\mathrm{HbA1c}$, glycated hemoglobin;BMI, body mass index; SBP, systolic blood pressure; DBP, diastolic blood pressure; TC,total cholesterol; LDL-C, low-density lipoprotein cholesterol; HDL-C, high-density lipoprotein cholestero; CAC-P, coronary artery calcium score progression. The data presented are the means \pm standard deviations, median (Q1-Q3) or numbers (percentages). 


\begin{tabular}{|c|c|c|c|}
\hline \multirow[b]{2}{*}{ Never } & \multicolumn{3}{|c|}{ coronary artery calcium score progression } \\
\hline & $369(12.97 \%)$ & $723(14.90 \%)$ & \\
\hline Past drinker & $39(1.37 \%)$ & $88(1.81 \%)$ & \\
\hline Current drinker & $2436(85.65 \%)$ & $4041(83.29 \%)$ & \\
\hline Reflux esophagitis status, (n, \%) & & & 0.002 \\
\hline Negative & $2729(89.48 \%)$ & $4446(87.16 \%)$ & \\
\hline Positive & $321(10.52 \%)$ & $655(12.84 \%)$ & \\
\hline Hypertension, n( \%) & & & $<0.001$ \\
\hline Negative & $2878(94.36 \%)$ & $4642(91.00 \%$ & \\
\hline Positive & $172(5.64 \%)$ & $459(9.00 \%)$ & \\
\hline Diabetes, n( \%) & & & $<0.001$ \\
\hline Negative & $2987(97.93 \%)$ & 4925 (96.55\%) & \\
\hline Positive & $63(2.07 \%)$ & $176(3.45 \%)$ & \\
\hline Dyslipidemia, n( \%) & & & 0.012 \\
\hline Negative & $2876(94.30 \%)$ & $4737(92.86 \%$ & \\
\hline Positive & $174(5.70 \%)$ & $364(7.14 \%)$ & \\
\hline Ischemic heart disease, $n(\%)$ & & & 0.032 \\
\hline Negative & $3045(99.84 \%)$ & $5078(99.55 \%$ & \\
\hline Positive & $5(0.16 \%)$ & $23(0.45 \%)$ & \\
\hline Cerebrovascular disease $\mathrm{n}(\%)$ & & & 0.996 \\
\hline Negative & 3047 (99.90\%) & $5096(99.90 \%$ & \\
\hline Positive & $3(0.10 \%)$ & $5(0.10 \%)$ & \\
\hline $\begin{array}{l}\text { HbA1c, glycated hemoglobin;BMI } \\
\text { pressure; TC,total cholesterol; LD } \\
\text { lipoprotein cholestero; CAC-P, cor } \\
\text { means } \pm \text { standard deviations, me }\end{array}$ & $\begin{array}{l}\text { index; SBP, syst } \\
\text { sity lipoprotein c } \\
\text { calcium score pr } \\
\text { ) or numbers (pe }\end{array}$ & $\begin{array}{l}\text { ressure; DBP, d } \\
\text { HDL-C, high-der } \\
\text { The data prese }\end{array}$ & $\begin{array}{l}\text { lic blood } \\
\text { are the }\end{array}$ \\
\hline
\end{tabular}

To test the robustness of our results, we performed a sensitivity analysis. We converted HbA1c into a categorical variable according to the quartile, and calculated the $\mathrm{P}$ for trend in order to verify the results of $\mathrm{HbA} 1 \mathrm{c}$ as the continuous variable, and to examine the possibility of nonlinearity. 
Modeling was performed with the statistical software packages R (http://www.R-project.org, The R Foundation) and EmpowerStats (http://www. empowerstats.com, X\&Y Solutions, Inc, Boston, MA). P values less than 0.05 (two-sided) were considered statistically significant.

\section{Results}

\section{Baseline characteristics of participants}

The baseline characteristics of these included participants were listed in Table 1. The average age was $53.82 \pm 7.66$ years old and about $90.98 \%$ were male. The incidence of progression was $62.58 \%$ (5101/8151). For all variables in Table 1, except cerebrovascular disease, there were statistically significant differences between the no progression group and the progression group ( $P$ values $<0.05)$. When compared with no progression group, HbA1c, age, sex, duration, BMI, weight, SBP, DBP, TC, LDL-C, triglycerides, smoking status, alcohol consumption, reflux esophagitis status, hypertension, diabetes, dyslipidemia, ischemic heart disease and cerebrovascular disease increased significantly in the progression group, while the opposite results were detected in covariates in terms of height and HDL-C.

\section{The results of multivariate analyses using Cox proportional-hazards regression model}

To evaluate the correlation between $\mathrm{HbA} 1 \mathrm{c}$ and coronary artery calcium score progression, three models (Cox proportional-hazards regression model) were constructed(Table 2). In un-adjusted model, an increase of $1 \%$ of $\mathrm{HbA} 1 \mathrm{c}$ was related with $24 \%$ increases of risk of coronary artery calcium score progression ( $\mathrm{HR}=1.24,95 \% \mathrm{Cl}: 1.20$ to 1.27$)$. The results was statistically significant. In minimallyadjusted model, when we only adjusted for demographic variables, each additional $1 \%$ of $\mathrm{HbA} 1 \mathrm{c}$ increases by $23 \% \square \mathrm{HR}=1.23,95 \% \mathrm{Cl}: 1.19$ to 1.26 ). In fully-adjusted model, Each additional $1 \%$ of $\mathrm{HbA} 1 \mathrm{c}$ was accompanied by a $22 \%$ increases in coronary artery calcium score progression $(H R=1.22$, $95 \% \mathrm{Cl}: 1.18$ to 1.26$)$. The distribution of confidence intervals indicated that the link between $\mathrm{HbA} 1 \mathrm{c}$ and coronary artery calcium score progression obtained by the model was reliable. 
Table 2

Univariate and multivariate analyses of the association between $\mathrm{HbA} 1 \mathrm{c}$ and coronary artery calcium score progression

\begin{tabular}{|llll|}
\hline Variable & $\begin{array}{l}\text { Non-adjusted model } \\
\text { HR }(95 \% \mathrm{Cl})\end{array}$ & $\begin{array}{l}\text { Minimally-adjusted model } \\
\text { HR }(95 \% \mathrm{Cl})\end{array}$ & $\begin{array}{l}\text { Fully-adjusted model } \\
\text { HR }(95 \% \mathrm{Cl})\end{array}$ \\
\hline HbA1c $(\%)$ & $1.24(1.20,1.27)$ & $1.23(1.19,1.26)$ & $1.22(1.18,1.26)$ \\
\hline HbA1c group & & & 1.0 \\
\hline Q1 & 1.0 & 1.0 & $1.42(1.29,1.56)$ \\
\hline Q2 & $1.33(1.21,1.45)$ & $1.32(1.20,1.44)$ & $1.63(1.48,1.80)$ \\
\hline Q3 & $1.61(1.47,1.76)$ & $1.57(1.44,1.72)$ & $1.98(1.80,2.17)$ \\
\hline Q4 & $2.01(1.85,2.19)$ & $1.92(1.77,2.10)$ & $<0.0001$ \\
\hline P for trend & $<0.0001$ & $<0.0001$ & \\
\hline HR, Hazards ratio; Cl, confidence interval; HbA1c, glycated hemoglobin & \\
\hline Non-adjusted model: we do not adjusted for any variates & \\
\hline Minimally-adjusted model: only sex and age are adjusted for & \\
\hline Fully-adjusted model: all variates presented in Table 1 are adjusted for & \\
\hline
\end{tabular}

The nonlinearity addressing by Cox proportional hazards regression model with cubic spline functions

Through the Cox proportional hazards regression model with cubic spline functions and smooth curve fitting, we observed that the correlation between $\mathrm{HbA} 1 \mathrm{c}$ and coronary artery calcium score progression was nonlinear (Fig. 2). For that reason, data were fit to a piecewise Cox proportional-hazards regression model to fit two different slopes. Based on the sensitivity analysis, we also fit data by standard Cox proportional-hazards regression model, and selected the best fit model through log-likelihood ratio test (Table 3 ). In our study, the $P$ for log-likelihood ratio test was less than 0.05 , we hence used two-piecewise model to fitting the association between $\mathrm{HbA} 1 \mathrm{c}$ and coronary artery calcium score progression. By recursive algorithm, we first obtained the inflection point was 5.8, and then calculated the effect sizes and confidence interval on the left and right of the inflection point by two-piecewise Cox proportional-hazards regression model. On the left side of inflection point, the effect size and $95 \% \mathrm{Cl}$ were $2.05,1.85$ to 2.27 , respectively. On the right side of inflection point, the effect size and $95 \% \mathrm{Cl}$ were $1.04,0.99$ to 1.10 , respectively. 
Table 3

Nonlinearity explaination on $\mathrm{HbA} 1 \mathrm{c}$ and coronary artery calcium score progression using two-piecewise linear model

\begin{tabular}{|ll|}
\hline & Effect size $(95 \% \mathrm{Cl})$ P value \\
\hline Fitting model by standard linear regression & $1.22(1.18,1.26)<0.0001$ \\
\hline Fitting model using two-piecewise linear model & \\
\hline Inflection point & 5.8 \\
\hline$<5.8$ & $2.05(1.85,2.27)<0.0001$ \\
\hline$>5.8$ & $1.04(0.99,1.10) 0.1123$ \\
\hline P for log-likelihood ratio test & $<0.001$ \\
\hline The adjustment strategy is the same with fully-adjusted model \\
\hline
\end{tabular}

\section{Discussion}

In this observational retrospective cohort study, we found a nonlinear saturation effect between HbA1c and the progression of coronary artery calcium score. When $\mathrm{HbA} 1 \mathrm{c}$ was less than 5.8 , there was a positive correlation between $\mathrm{HbA} 1 \mathrm{c}$ and the progression of coronary artery calcium score, while above 5.8, this positive correlation tended to be saturated. Even if $\mathrm{HbA} 1 \mathrm{c}$ increased again, the risk of coronary artery calcium score progression would not increase.

Some previous studies have shown the consistent results with ours investigating correlation between $\mathrm{HbA} 1 \mathrm{c}$ and coronary artery calcium score progression or coronary atherosclerosis. Based on 411 patients without history of known diabetes mellitus, Reza Ajudani reported that $\mathrm{HbA} 1 \mathrm{c}$ may be an independent diagnostic factor in non-diabetic patients with severe coronary atherosclerosis in a cross-sectional study[19]. Besides, Wenhui Zhao et al. used African American and white diabetic patients as the study population and obtained similar results[20]. Rivera JJ et al. reached similar conclusions in 1043 asymptomatic people without diabetes[21]. In addition, in veterans affairs diabetes trial participants, Peter D Reaven et al. found that although intensive hypoglycemic therapy did not reduce cardiovascular events in the study cohort as a whole, intensified hypoglycemic therapy could reduce cardiovascular events in people with less severe coronary atherosclerosis[22]. However, it is not that lower HbA1c level is associated with better the occurrence and outcome of cardiovascular events. Hertzel C Gerstein et al. reported that using intensive therapy to position glycosylated hemoglobin levels below $6.0 \%$ could increase mortality, but did not significantly reduce major cardiovascular events in type 2 diabetes[23]. The inconsistency of these studies may be due to the small sample size, different study population, different forms of outcome variables and few cases of coronary heart disease, which may limit statistical power.

Our study has some strengths, and we listed it as follows. First, the sample size of 
this study was relatively large. Seconde, previous articles that also studied HbA1c and coronary artery calcium score or coronary atherosclerosis did not clarify the nonlinear relationship[19, 20, 24-29]. To the best of our knowledge, this is the first time that a non-linear relationship has been reported and an inflection point has been determined and explained in exploring the correlation between $\mathrm{HbA} 1 \mathrm{c}$ and coronary artery calcium score progression. Third, this study is an observational study and therefore susceptible to potential confounding. We used strict statistical adjustment to minimize residual confounders. Forth, we ensured the robustness of the results through sensitivity analysis(conversion of target independent variable form). This makes our results more reliable.

Our research has the following shortcomings and needs attention. Firstly, our findings can be generalized to persons who had undergone physical examinations at South Koreaa only. Secondly, due to the limitation of the original data, we could not observe the correlation between $\mathrm{HbA} 1 \mathrm{c}$ and the progress of coronary artery calcium score in diabetes and prediabetes patients. Finally, as in all observational studies, even though known potential confounders factors were controlled for, there might have been still uncontrolled confounders.

\section{Conclusion}

The relationship between $\mathrm{HbA} 1 \mathrm{c}$ and coronary artery calcium score progression is non-linear. $\mathrm{HbA} 1 \mathrm{c}$ is positively related with coronary artery calcium score progression when $\mathrm{HbA} 1 \mathrm{c}$ was less than $5.8 \%$.

\section{Abbreviations}

HbA1c: glycated hemoglobin; CHD: coronary heart disease; BMI: body mass index; SBP: systolic blood pressure; DBP: diastolic blood pressure; TC: total cholesterol; LDL-C: low-density lipoprotein cholesterol; HDL-C: high-density lipoprotein cholestero; EGD: esophagogastroduodenoscopy; CT: coronary computed tomography

\section{Declarations}

\section{Acknowledgements}

The author is very grateful to the data providers of the study. They completed the entire study. They are (the rankings and institutions of these researchers were ranked according to the" reference [17]" ) Yang Won Min, Byeong Geun Song, Hyuk Lee, Byung-Hoon Min, Jun Haeng Lee, Hee Jung Son, Poong-Lyul Rhee(corresponding author), Jae J. Kim, (Department of Medicine, Samsung Medical Center, Sungkyunkwan University School of Medicine, Seoul, South Korea), Hye Seung Kim and Kyunga Kim (Biostatistics and Clinical Epidemiology Center, Samsung Medical Center, Seoul, South Korea). The authors also thank Chi Chen of Yi-er college for data processing and statistical analysis.

\section{Authors' contributions}


JY contributed to the drafting of the manuscript, and analysis and interpretation of the data. BG contributed to the conception and critical revision of the manuscript, analysis and interpretation of the data and approved the final version of the submitted manuscript. Both authors read and approved the final manuscript.

\section{Funding}

The authors received no specific funding for this work.

\section{Availability of data and materials}

Data can be downloaded from the "PLOS ONE" database (https://journals.plos.org/plosone/).

\section{Ethics approval and consent to participate}

In the previously published article [17], Yang Won Min, et al. has clearly stated that: the study was conducted in accordance with the Declaration of Helsinki. Informed consent was obtained from all Participants.

\section{Consent for publication}

Not applicable.

\section{Competing interests}

The authors declare that they have no competing interests.

\section{Author details}

*Correspondence: gygb2004@163.com

${ }^{1}$ Department of Imaging Diagnostics, Guizhou Medical University, 9 Beijing Road, Yunyan District, Guiyang, 550001, China.

\section{References}

[1] American Diabetes Association. Diagnosis and classification of diabetes mellitus. Diabetes Care. 2010;33 Suppl 1:S62-9.

[2] Ding N, Kwak L, Ballew SH, et al. Traditional and nontraditional glycemic markers and risk of peripheral artery disease: The Atherosclerosis Risk in Communities (ARIC) study. Atherosclerosis. 2018;274:86-93.

[3] Sacks DB, Arnold M, Bakris GL, et al. Guidelines and recommendations for laboratory analysis in the diagnosis and management of diabetes mellitus. Diabetes Care. 2011;34(6):e61-99. 
[4] Selvin E, Steffes MW, Zhu H, et al. Glycated hemoglobin, diabetes, and cardiovascular risk in nondiabetic adults. N Engl J Med. 2010;362(9):800-11.

[5] Andersson C, Johnson AD, Benjamin EJ, et al. 70-year legacy of the Framingham Heart Study. Nat Rev Cardiol. 2019;16(11):687-698.

[6] Go AS, Mozaffarian D, Roger VL, et al. Heart disease and stroke statistics--2014 update: a report from the American Heart Association. Circulation. 2014;129(3):e28-e292.

[7] Sarah Rosner P, Pencina MJ, Shih-Jen $\mathrm{H}$, et al. Trends in cardiovascular disease risk factors in individuals with and without diabetes mellitus in the framingham heart study. Circulation. 2009;120(3):212-20.

[8] Kang DO, Seo HS, Choi BG, et al. Absolute change in fasting plasma glucose over 12 months is associated with 2-year and 5-year major adverse cardiovascular events in patients with drug-eluting stent implants. Int J Cardiol. 2015;179:146-52.

[9] Lin B, Koibuchi N, Hasegawa Y, et al. Glycemic control with empagliflozin, a novel selective SGLT2 inhibitor, ameliorates cardiovascular injury and cognitive dysfunction in obese and type 2 diabetic mice. Cardiovasc Diabetol. 2014;13:148.

[10] Liu XJ, Wan ZF, Zhao N, et al. Adjustment of the GRACE score by HemoglobinA1c enables a more accurate prediction of long-term major adverse cardiac events in acute coronary syndrome without diabetes undergoing percutaneous coronary intervention. Cardiovasc Diabetol. 2015;14:110.

[11] Ashraf $\mathrm{H}$, Boroumand MA, Amirzadegan A, et al. Hemoglobin A1C in non-diabetic patients: an independent predictor of coronary artery disease and its severity. Diabetes Res Clin Pract. 2013;102(3):225-32.

[12] Chen CL, Yen DH, Lin CS, et al. Glycated hemoglobin level is an independent predictor of major adverse cardiac events after non fatal acute myocardial infarction in nondiabetic patients: a retro spective observational study. Medicine (Baltimore). 2017;96(18):e6743.

[13] Açar B, Ozeke O, Karakurt M,et al. Association of Prediabetes With Higher Coronary Atherosclerotic Burden Among Patients With First Diagnosed Acute Coronary Syndrome. Angiology. 2019;70(2):174-180.

[14] Scicali R, Giral P, Gallo A, et al. HbA1c increase is associated with higher coronary and peripheral atherosclerotic burden in non diabetic patients. Atherosclerosis. 2016;255:102-8.

[15] Blake GJ, Pradhan AD, Manson JE, et al. Hemoglobin A1c level and future cardiovascular events among women. Arch Intern Med. 2004;164(7):757-61.

[16] ADVANCE Collaborative Group, Patel A, MacMahon S, et al. Intensive blood glucose control and vascular outcomes in patients with type 2 diabetes. N Engl J Med. 2008;358(24):2560-72. 
[17] Min YW, Song BG, Kim HS, et al. Associations between reflux esophagitis and the progression of coronary artery calcification: A cohort study. PLoS One. 2017;12(10):e0184996.

[18] Agatston AS, Janowitz WR, Hildner FJ, et al. Quantification of coronary artery calcium using ultrafast computed tomography. J Am Coll Cardiol. 1990;15(4):827-32.

[19] Ajudani R, Rezaee-Zavareh MS, Karimi-Sari H, et al. Glycosylated haemoglobin and coronary atherosclerosis in non-diabetic patients: is it a prognostic factor? Acta Cardiol. 2017;72(5):522-528.

[20] Zhao W, Katzmarzyk PT, Horswell R, et al. HbA1c and coronary heart disease risk among diabetic patients. Diabetes Care. 2014;37(2):428-35.

[21] Rivera JJ, Choi EK, Yoon YE, et al. Association between increasing levels of hemoglobin A1c and coronary atherosclerosis in asymptomatic individuals without diabetes mellitus. Coron Artery Dis. 2010;21(3):157-63.

[22] Reaven PD, Moritz TE, Schwenke DC,et al. Intensive glucose-lowering therapy reduces cardiovascular disease events in veterans affairs diabetes trial participants with lower calcified coronary atherosclerosis. Diabetes. 2009;58(11):2642-8.

[23] Action to Control Cardiovascular Risk in Diabetes Study Group, Gerstein HC, Miller ME, et al. Effects of intensive glucose lowering in type 2 diabetes. N Engl J Med. 2008;358(24):2545-59.

[24] Svanteson M, Holte KB, Haig Y, et al. Coronary plaque characteristics and epicardial fat tissue in long term survivors of type 1 diabetes identified by coronary computed tomography angiography. Cardiovasc Diabetol. 2019;18(1):58.

[25] Lindholt JS, Frystyk J, Hallas J, et al. Feasibility Study of Advanced Cardiovascular Screening in Middle-Aged Patients with Diabetes. Clin Epidemiol. 2020;12:447-455.

[26] Jung CH, Lee WY, Kim SY, et al. The relationship between coronary artery calcification score, plasma osteoprotegerin level and arterial stiffness in asymptomatic type 2 DM. Acta Diabetol. 2010;47 Suppl $1: 145-52$.

[27] Yang J, Zhou Y, Zhang T, et al. Fasting Blood Glucose and HbA1c Correlate With Severity of Coronary Artery Disease in Elective PCI Patients With HbA1c 5.7\% to 6.4. Angiology. 2020;71(2):167-174.

[28] Rhee EJ, Cho JH, Kwon H, et al. Association Between Coronary Artery Calcification and the Hemoglobin Glycation Index: The Kangbuk Samsung Health Study. J Clin Endocrinol Metab. 2017;102(12):4634-4641.

[29] Huo X, Gao L, Guo L, et al. Risk of non-fatal cardiovascular diseases in early-onset versus late-onset type 2 diabetes in China: a cross-sectional study. Lancet Diabetes Endocrinol. 2016;4(2):115-24. 


\section{Figures}

Yang Won Min et al. collectting participants who were $\geq 20$ years old and underwent health check-up programs at the Center for Health Promotion of Samsung Medical Center from March 2003 to December 2013 ( N=199,375)

$\downarrow$\begin{tabular}{l}
$\begin{array}{l}\text { Exclusions }(\mathbf{n}=\mathbf{1 7 2}, \mathbf{0 2 7}) \\
\text { No EGD and coronary CT scan at the same } \\
\text { visit }\end{array}$ \\
\hline
\end{tabular}

Yang Won Min et al. collectting participants who were $\geq 20$ years old and underwent EGD and coronary CT scan at the same visit at the Center for Health Promotion of Samsung Medical Center from March 2003 to December 2013( $n=27,348)$

\begin{tabular}{|l|}
\hline \\
Exclusions $(\mathbf{n}=\mathbf{1 9 , 1 9 7 )}$ \\
-No follow up coronary CT scan $(\mathrm{n}=19,038)$ \\
-Missing data $(\mathrm{n}=89)$ \\
-Missing HbA1c at the first physical \\
examination $(\mathrm{n}=70)$
\end{tabular}

\section{Study participants}

Participants who were $\geq 20$ years old and underwent EGD and coronary CT scan at the same visit and a follow up coronary $\mathrm{CT}$ scan without missing data and missing $\mathrm{HbA1c}$ at the first physical examination at the Center for Health Promotion of Samsung Medical Center from March 2003 to December 2013( $n=8151)$

\section{Figure 1}

Study flow chart. EGD, esophagogastroduodenoscopy; CT, computed tomography 


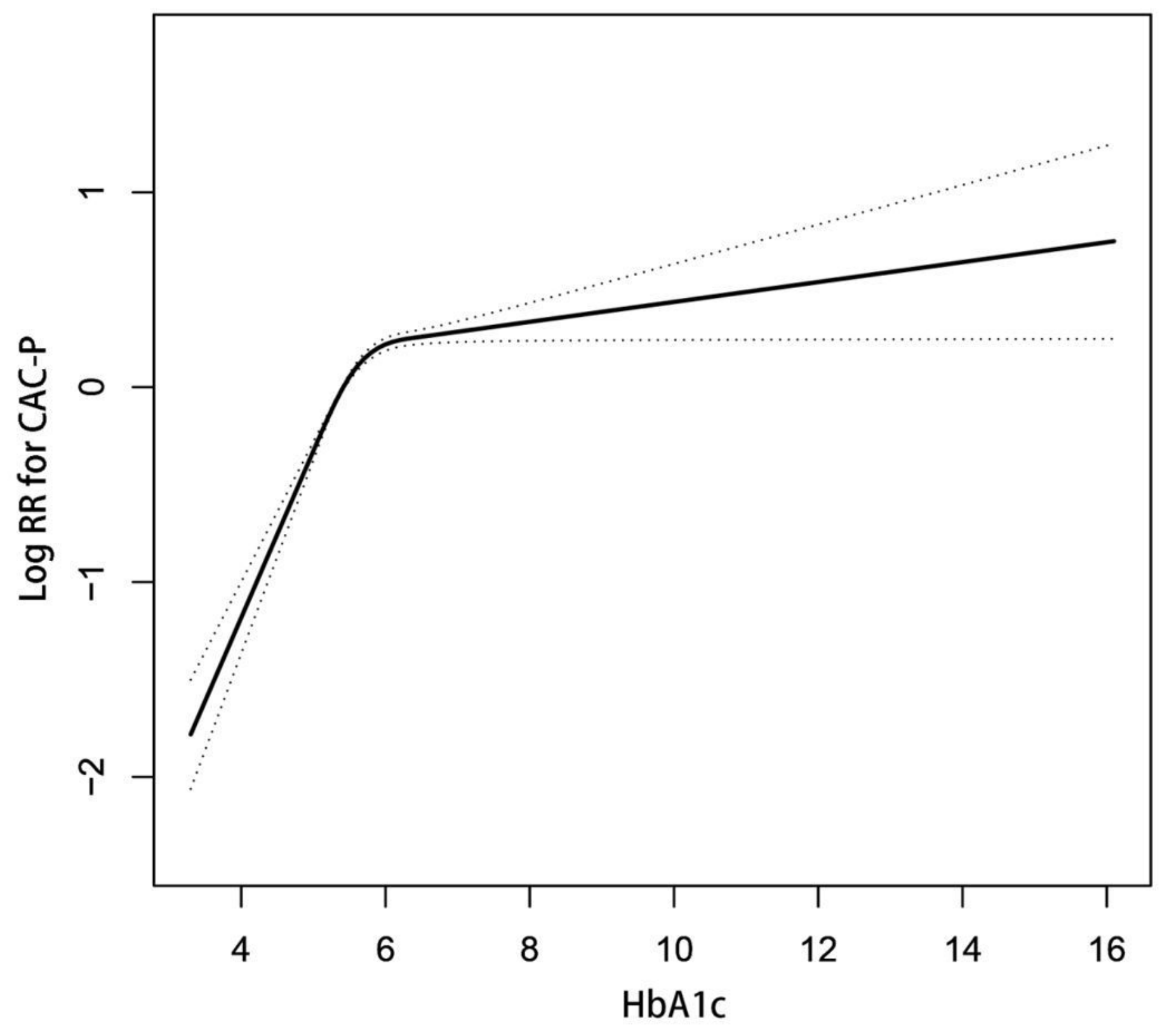

Figure 2

Relationship between $\mathrm{HbA} 1 \mathrm{c}$ and coronary artery calcium score progression by using Lowess smoothing technique. HbA1c, glycated hemoglobin; CAC-P,coronary artery calcium score progression 